\title{
Sépharades et Ashkénazes dans l'œuvre romanesque d'Albert Cohen
}

\author{
Véronique Maisier \\ Southern Illinois University, Carbondale
}

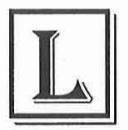

a sépharadité d'Albert Cohen, auteur francophone né dans la communauté juive de Corfou en 1895 à l'origine de nationalité ottomane puis naturalisé suisse en 1919, semble être source d'ambiguïté et occasionne différentes lectures. Lorsqu'Albert Bensoussan affirme qu' " [i]l y a inévitablement dans l'œuvre de Cohen une revendication de la séfaradité, ce qu'il appelait, lui, l'Orient juif »(1993, 131), il s'oppose à Denise Goitein-Galperin selon laquelle, "Cohen ne s'est jamais voulu ni déclaré porteur de ce que le jargon à la mode appelle 'sépharadité' » $(1989,217)$. Devant la différence d'opinion de ces deux experts de l'œuvre et de la pensée cohéniennes, les rapports de cet auteur à la sépharadité tels qu'ils s'expriment dans son écriture romanesque méritent d'être analysés et approfondis. À cette fin, il sera utile d'examiner la sépharadité des personnages appelés les Valeureux de France et d'étudier le rôle des Juifs ashkénazes également présents dans cette œuvre, avant d'analyser les diverses facettes des rapports sépharades-ashkénazes disséminées dans les romans cohéniens.

L'expression " Juif sépharade » regroupe traditionnellement les Juifs qui vécurent en Espagne après l'expulsion de Jérusalem. Dans son livre Les Juifs sépharades, Victor Malka distingue les communautés sépharade et ashkénaze dans les termes suivants :

C'est à partir du milieu du XIVe siècle que l'on a pris l'habitude de désigner les juifs vivant en Espagne comme des juifs sépharades, la communauté ashkénaze étant constituée, elle, par les descendants de ceux qui, exilés de Babylonie et de Palestine, se sont installés en Europe centrale et orientale. Depuis la fin du Moyen-Âge, ces communautés parlent communément un dialecte allemand tandis 
que les sépharades s'expriment en judéo-espagnol et, plus tard en judéo-arabe. $(1991,4)$

À travers l'œuvre romanesque de Cohen, nombreuses sont les références aux origines des personnages, aussi bien des cinq membres du groupe des Valeureux que du protagoniste principal Solal. Les Valeureux, Juifs de la Méditerranée et du soleil, proclament fièrement leur lignée et se déclarent les " descendants des Juifs d'Espagne à cheval qui furent vêtus de soie et portèrent dagues, rubans, roses et épées " $(1965,260)$. Ils vivent à Céphalonie depuis que la branche cadette de la famille des Solal s'est installée dans le ghetto de cette île grecque de la mer Ionienne au XVIII e siècle tandis que les membres de la branche aînée, " originaires d'Espagne, étaient venus à Céphalonie au début du XVI e siècle " $(1958,34)$. Leurs origines permettent à Bensoussan de décrire ces personnages comme "d'authentiques Sépharades, car descendants, d'une part, des communautés espagnoles d'autrefois - celles qui furent effacées par le décret d'expulsion de 1492-d'autre part, des Juifs venus de France et qui venaient eux-mêmes, probablement de cette même Espagne " $(1979,397)$.

Lors de leurs multiples voyages, les protagonistes céphaloniens rencontrent fréquemment des Juifs ashkénazes qu'ils décrivent comme des "Juifs polonais de jargon de malheur, mangeurs de carpes froides ", "Juifs de la froidure, nés de l'accouplement du singe et du pou volant de Lituanie " $(1965,261)$. L'opposition sépharade versus ashkénaze se trouve maintes fois soulignée par les Valeureux, et en particulier par Mangeclous qui se demande : "Ces Russes, qu'ont-ils de commun avec moi que je suis un Juif du soleil? » $(1958,444)$. Selon Catherine Milkovitch-Rioux, « [la] distinction [entre Sépharades et Ashkénazes] est établie essentiellement sur l'opposition entre le soleil et la froidure » $(1995,351)$. Cependant, la distinction, plus profonde, repose également sur des critères historiques, linguistiques, géographiques, culturels et même physiologiques entre les Juifs sépharades et ashkénazes, distinctions multiples soulignées tout au long de l'œuvre qui tendent dans un premier temps à revendiquer la supériorité des origines sépharades et dans un deuxième temps à condamner les attributs tout négatifs des Ashkénazes.

Cependant, les critiques véhémentes des Valeureux envers les Juifs ashkénazes ne sauraient être lues au pied de la lettre d'un écrivain pour lequel la vérité est " ce qui est entre les mots " $(1958,53)$. Le rejet des Ashkénazes parât trop flagrant pour être pris sérieusement chez un auteur qui force la caricature chez ses personnages, «tel l'amateur d'épices qui en met beaucoup qui en met trop et à s'en emporter la bouche pour les

\section{4 * LittéRéalité}


savourer davantage » $(1986,899)$. Cohen développe en effet dans ses écrits une stratégie de dénonciation des stéréotypes antisémites par l'exagération grotesque des personnages juifs, ce qui lui permet de reprendre ces stéréotypes à son propre compte et de les manipuler, de les utiliser afin d'en changer le sens et la portée. Cette technique de réappropriation donne naissance aux Valeureux sépharades, et il est raisonnable de penser qu'elle est également à l'origine des descriptions a priori dégradantes des Juifs ashkénazes.

La tendresse marquée du narrateur cohénien envers Jérémie, malgré la caricature de ce représentant du judaïsme ashkénaze, étaye cette théorie. La rencontre entre les Valeureux et Jérémie, "sorti récemment d'une prison allemande " $(1965,196)$, se fait par l'intermédiaire d'un Gentil prénommé Scipion, petit marseillais que Mangeclous, l'un des Valeureux a rencontré alors qu'ils faisaient tous deux leur service militaire. L'amitié de longue haleine qui unit ces personnages sur fond de prison, d'armée et de guerre, montre que les trois groupes, Sépharades, Ashkénazes et Gentils peuvent vivre harmonieusement malgré le contexte socio-historique. Il existe un parallélisme intéressant entre l'œuvre romanesque et la vie de l'auteur puisque le Scipion de Marseille retrouve le Juif ashkénaze fugitif Jérémie à Genève, de même qu'Albert Cohen, venu de Marseille, découvre le judaïsme ashkénaze à Genève, lorsqu'il rencontre de nombreux Juifs russes immigrés qui ont fui les pogromes dans les années 1914-1920. Peu de choses sont connues sur Cohen à cette époque de sa vie, lorsque, jeune étudiant à Genève, il rencontre de nombreux réfugiés, parmi lesquels la population russe est importante. Gérard Valbert souligne néanmoins l'importance de cette période : "Pour saisir le cheminement de l'écrivain, il ne faut pas négliger cette rencontre des Russes ", et va jusqu’à affirmer que "Les Russes vont hanter le jeune homme » (1990, 90-92). Cohen devient alors conscient des pogromes et des persécutions menés contre le peuple juif ashkénaze :

La distance n'empêche pas la sympathie et Cohen se renseignera sur des événements que les journaux d'Occident avaient escamotés. [...] À l'époque, il avait eu connaissance de témoignages, de documents, d'enquêtes. Les dossiers du drame, il les avait compulsés et ils alimentaient de curieuses histoires. [...] Il avait beaucoup lu aussi et parfois, contant la tragédie des pogromes, il vous récitait des pages des frères Tharaud qu'avait retenues sa mémoire. (Valbert 1990, 92-93) 
La sympathie de Cohen pour la situation des Juifs russes est manifeste dans ses écrits, et en particulier dans le portrait de Jérémie, créé pour la pièce Ezéchiel dès 1930 et davantage développé dans le roman Mangeclous en 1938. Ce « représentant du peuple élu » $(1965,194)$, né en Lituanie mais dont la nationalité ne peut être déterminée avec certitude, chancelle sur des bottines de femme et se chatouille sous les bras pour se faire rire le jour de sabbat selon la tradition hassidique. S'il se délecte effectivement de l'inévitable carpe froide et devient selon Mangeclous " cet imbécile de Juif polonais » (215), la distinction sépharade versus ashkénaze se trouve brouillée par l'amitié qui existe entre ces personnages.

Plus encore, une lecture approfondie de l'œuvre cohénienne révèle que la séparation entre l'Orient et l'Occident ne saurait être nette chez cet auteur. Philippe Zard remarque fort justement qu'il est impossible « de voir dans l'œuvre un lieu d'affrontement entre deux sphères culturelles hétérogènes. L'Orient de Cohen est tout entier pétri de culture, de souvenirs, de références occidentales, tout comme l'Occident [...] est irrigué, à son corps défendant, de sources orientales. [...] les termes d'" Orient " et d' "Occident " ne désignent pas des ensembles culturels purs, mais deux combinaisons de valeurs hybrides [...]» $(1999,17)$. La présence de ces deux sphères culturelles dans la pensée de l'auteur donne naissance à une œuvre dont l'hybridité incite et justifie à la fois le parallèle fréquemment établi entre Cohen et les auteurs ashkénazes. Catherine Milkovitch-Rioux affirme que les Valeureux « sont autant d'incarnations du schlemibl, les représentants de l'humour juif » et qu'on " peut également rapprocher les personnages céphaloniens, en particulier Mangeclous, des Schnorrers, ces mendiants juifs exaltés par Zangwill, maîtres dans l'art de renverser les rôles » $(1995,383)$. De même, Arnold Mandel affirme que : "Leur île de Céphalonie est la jumelle méditerranéenne du Kassrilevké judéo-slave de Cholem Aleïchem. C'est donc un rond-point géographique qui, bien que délimité et impressionné par son climat spécifique, se retrouve et se reproduit à distance, sous d'autres latitudes » $(1968,47)^{1}$.

La propension de Mangeclous à imaginer toutes sortes de métiers le rapproche du personnage familier de la littérature ashkénaze appelé

1 Mentionnons cependant les commentaires d'Elaine Marks qui affirme au contraire : «Albert Cohen's baroque and picaresque style makes his Jews very different from the more traditional representations of Ashkenazic Jews sensitive, brilliant, persecuted, sorrowful - to which novels of stories written between the Dreyfus case and the aftermath of the Shoah have accustomed us » $(1996,68)$. 
luftmensch ${ }^{2}$. Les frasques mangeclousiennes évoquent cet homme dont le nom signifie littéralement « homme de l'air » qui :

se déplace aussi comme l'air, en se laissant emporter par les ailes de son imagination et par son flair qu'il croit infaillible et qui le pousse sans cesse vers des affaires plus mirobolantes et plus incertaines les unes que les autres. (Stora-Sandor 1984, 137-138)

Ce luftmensch des pays de l'Europe de l'Est ${ }^{3}$ n'est pas inconnu de Cohen qui utilise ce terme dans un rapprochement révélateur lors d'un entretien accordé à Victor Malka en 1981 : «Si j'aime Mangeclous, c'est à cause de la tendresse que j'ai pour un pauvre Luftmenschen. Je les aime ceux-là " (21). L'auteur qui connaît les " innombrables histoires juives, anonymement surgies au sein des collectivités opprimées et malheureuses de l'Est européen " (Rabi 1963, 36) importe certains aspects de cette culture et les adapte à la communauté sépharade des Valeureux. Ces " innombrables histoires " de l'Europe de l'Est s'ajoutent et se fondent parmi les " interminables [...], infinies histoires douloureuses ou bouffonnes $\mathrm{du}$ ghetto " $(1954,92)$ de Corfou que sa mère lui raconte. Le ghetto juif de Céphalonie devient alors une sorte d'analogue oriental du shtetl, c'est-à-dire de la bourgade juive décrite par Arnold Mandel dans les termes suivants :

Le lieu de résidence le plus caractéristique des Juifs de l'Est européen, la commune juive par excellence, c'était ce que l'on appelle en yiddish, le shtetl, la toute petite ville ou la bourgade, ne se distinguant du simple village que parce qu'il était généralement un lieu où se tenait un marché hebdomadaire et donc avait une 'place du marché', et présentait une certaine animation économique. $(1974,31)$

2 Wladimir Rabi écrit à ce sujet : "Quant à Mangeclous, héros central au milieu de ses Valeureux, il est vraiment le schlemiel du folklore yiddish ». Rabi n'établit pas de différence autre que chronologique entre le schlemiel et le luftmensch: "Mais qu'est-ce que le schlemiel? C'est le personnage issu de la société psycho-économique du chtettel, le "luftmensch " du Xix ${ }^{e}$ siècle européen » $(1972,73)$.

3 Salo Baron, dans son livre The Russian Jew Under Tsars and Soviets, écrit : "It has been established that in many communities up to 40 percent of the entire Jewish population consisted of families of so-called Luftmenschen, that is persons without any particular skills, capital, or specific occupations » $(1964,114)$. 
Mandel établit une distinction intéressante entre le shtetl et le ghetto, le premier étant une zone de peuplement dans laquelle les habitants, à majorité Juifs, connaissent un régime d'autonomie interne, sont libres de leurs mouvements et vivent proches de la nature, alors que le second est un " espace muré en plein cour de la ville chrétienne " dans lequel "les habitants [...] sont retranchés et enfermés. Ils vivent dans des venelles sombres et n'ont aucun contact avec la nature " (1974, 32-33). Le terme de ghetto juif est le seul utilisé afin de décrire le lieu de résidence des Valeureux à Céphalonie. La population juive représente une minorité sur l'île constituée majoritairement de Chrétiens, dont elle est séparée par des chaînes : "le ghetto de hautes maisons eczémateuses que des chaînes séparaient de la douane et du port... " $(1958,17)$. Cependant, ce ghetto céphalonien ne paraît pas emprisonner ses habitants qui vivent au soleil, se déplacent librement et gardent accès à la nature. Ainsi, Saltiel et Solal se promènent dans la "forêt argentée d'oliviers » $(1958,16)$, Mangeclous fait des petits tours du côté de la mer et Salomon, dans un moment de joie intense, galope avec " la frénésie du poulain » $(1965,111)$ à travers forêts et prairies avant de se jeter dans la mer. Les descriptions céphaloniennes évoquent bien davantage un shtetl qu'un ghetto dans lequel vont à leurs affaires les luftmenschen, les shnorrers, et les schlemiel cohéniens.

Le rejet très - trop — visible de la communauté ashkénaze défie le lecteur d'accepter les termes de l'auteur et le pousse à faire la part du vrai entre les provocations, l'ironie et les remises en cause. Plutôt que d'une revendication de la sépharadité, ou même que d'un rapprochement souhaité entre Sépharades et Ashkénazes, il nous semble que c'est le mélange, l'hybridité culturelle que l'auteur promeut dans son ouvre qu'il veut « large, et, comme la mer, accueillante à tous » (Goitein-Galperin 1987, 13).

Dans son roman Belle du Seigneur, l'épisode onirique de « Rosenfeld et compagnie » (1986, 895-900) synthétise un œcuménisme rêvé entre Juifs de l'Est et Juifs du Sud dans la description d'une tribu aux particularismes fortement multiculturels. La famille Rosenfeld et ses différents membres constituent un amalgame de cultures représenté par le multilinguisme des uns et des autres qui communiquent en russe, en anglais, en espagnol et en hébreu. Benjamin, l'un des fils Rosenfeld « chante une prière hébraïque suivie d'une mélodie populaire suisse d'une danse russe et d'une fable non demandée [de La Fontaine] » (1986, 890). Si les Rosenfeld viennent de Russie et sont Ashkénazes (comme le révèlent une nouvelle fois les références culinaires à quelques plats tels que les gâteaux roumains, les gefilte fisch ou les apfelstrudels), la communauté de l'expérience juive 
s'exprime indépendamment des origines dans un universalisme qui transforme graduellement les membres spécifiques de la famille (Benjamin âgé de six ans, la fille aînée, le beau-frère, etc.) en un bambin, un aïeul, diverses dames et même quelques « collatéraux obscurs » $(1986,893)$. Le rejet communautaire parât impossible, ce que souligne amplement Solal sépharade lorsqu'il intègre les Rosenfeld dans sa famille : "j'étais bien avec les Rosenfeld tout à l'heure j'étais en famille et avec les miens et je les chérissais » $(1986,899)$. Devant la répression, l'expulsion et l'extermination subies à travers les siècles, les Sépharades et les Ashkénazes ne forment qu'un peuple et partagent les " plaies d'un peuple persécuté bosses et plaies d'un peuple tordu par des siècles de tourments » $(1986,899)$. L'alliance des deux communautés juives se révèle dans une dernière référence culinaire. Tandis que dans l'épisode sioniste trouvé dans Solal, écrit dès 1930, Mangeclous avait souligné les différences en décrétant que les Ashkénazes étaient des concombres tandis que les Sépharades étaient le sel — « Ils sont les concombres et nous sommes le sel, dit énigmatiquement Mangeclous " $(1958,442)$ - dans Belle du Seigneur, écrit après la Shoah et plus significativement encore après la reconnaissance de l'État d'Israël, Rosenfeld se propose de partager des " concombres salés " (895) avec son hôte occidental afin de passer la " nuit à causer dans l'amitié ". Cette métaphore alimentaire proclame la possibilité d'une hybridité qui, préservant les particularités de chaque communauté, se trouverait renforcée - épicée - par ce que chacune peut offrir à l'autre.

\section{CEuvres citées}

Baron, Salo. The Russian Jew under Tsars and Soviets. New York : Macmillan, 1964.

Bensoussan, Albert. "L'image du Sépharade dans l'œuvre d'Albert Cohen ", Les Temps Modernes, no 394bis, 1979, 396-409.

Bensoussan, Albert. L'Échelle séfarade. Paris : L'Harmattan, 1993.

Cohen, Albert. Le Livre de ma mère. Paris : Gallimard, 1954.

Cohen, Albert. Solal. Paris : Gallimard, 1958.

Cohen, Albert. Mangeclous. Paris : Gallimard, 1965.

Cohen, Albert. Belle du Seigneur. Paris : Gallimard, 1986.

Cohen, Albert. "Le Juif et les romanciers français ", La Revue de Genève, $n^{\circ} 33,1923,340-351$.

Goitein-Galperin, Denise. "L'existence juive dans l'œuvre d'Albert Cohen ", Colloque "Les amours d'Albert Cohen ", 14 novembre 1987. Goitein-Galperin, Denise. "Albert Cohen revisité. La vision 
cohénienne de l'existence juive ", Revue des Études juives, n ${ }^{\circ}$ 198, 1989, 205-219.

Malka, Victor. "Albert Cohen : je suis un athée qui vénère Dieu ", Les Nouvelles Littéraires, no 2730, 1981, 21.

Malka, Victor. Les Juifs sépharades. Paris : Presses Universitaires de France, 1991.

Mandel, Arnold. «Albert Cohen ou l'ange Michaël », L'Arche, 1968, p. 4651.

Mandel, Arnold. La Vie quotidienne des Juifs hassidiques du 18e siècle à nos jours. Paris : Hachette, 1974.

Marks, Elaine. Marrano as Metaphor. The Jewish Presence in French Writing. Columbia University Press : New York, 1996.

Milkovitch-Rioux, Catherine. L'Univers mythique d'Albert Cohen. Personnages, décors et mise en scène. Villeneuve d'Ascq : Presses Universitaires du Septentrion, 1995.

Rabi, Wladimir. «L'univers d'Albert Cohen », L'Arche, no 184 , juin-juillet 1972, 73.

Stora-Sandor, Judith. L'Humour juif dans la littérature, de Job à Woody Allen. Paris : Presses Universitaires de France, 1984.

Valbert, Gérard, Albert Cohen, le seigneur. Paris, Grasset, 1990.

Wisse, Ruth. The Schlemiel as Modern Hero. Chicago : University of Chicago Press, 1971.

Zard, Philippe. La Fiction de l'Occident : Thomas Mann, Franz Kafka, Albert Cohen. Paris : Presses Universitaires de France, 1999. 